Nouvelles perspectives en sciences sociales

Revue internationale de systémique complexe et d'études relationnelles

\title{
Entre mesure, science et politique : construction et analyse d'un réseau international de copublications dans le domaine de l'éducation
}

\author{
Monique Dalud-Vincent et Romuald Normand
}

Volume 6, numéro 2, juin 2011

URI : https://id.erudit.org/iderudit/1005775ar

DOI : https://doi.org/10.7202/1005775ar

Aller au sommaire du numéro

Éditeur(s)

Prise de parole

ISSN

1712-8307 (imprimé)

1918-7475 (numérique)

Découvrir la revue

Citer cet article

Dalud-Vincent, M. \& Normand, R. (2011). Entre mesure, science et politique : construction et analyse d'un réseau international de copublications dans le domaine de l'éducation. Nouvelles perspectives en sciences sociales, 6(2),

197-232. https://doi.org/10.7202/1005775ar
Résumé de l'article

Notre étude vise à repérer certains réseaux d'experts et de chercheurs, à l'oeuvre à l'échelle internationale, qui produisent de nouveaux référentiels et de nouveaux instruments de mesure pour améliorer l'efficacité et la qualité de l'éducation. Pour ce faire, nous utilisons la méthodologie de l'analyse des réseaux (dont l'outil Pajek) afin d'objectiver et d'étudier les liens de co-publications. La définition de ces liens repose sur un travail préalable de constitution d'une base de références bibliographiques contenant 5300 références écrites par environ 3500 auteurs. On met alors en évidence des communautés épistémiques clairement identifiées et rattachées à un paradigme: des chercheurs de la school effectiveness, des économistes du capital humain, des psychométriciens spécialistes des comparaisons internationales de résultats. 


\title{
Entre mesure, science et politique : construction et analyse d'un réseau international de copublications dans le domaine de l'éducation
}

\author{
Monique Dalud-Vincent ${ }^{\mathrm{I}}$ \\ MEPS - Centre Max Weber, Université de Lyon, France \\ Romuald Normand \\ Institut Français de l'Éducation, École Normale Supérieure de Lyon, \\ France
}

B eaucoup de sociologues ont étudié les influences de la globalisation sur les politiques d'éducation à l'échelle internationale ${ }^{2}$. Ces chercheurs ont décrit minutieusement les

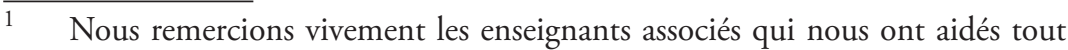
au long de ce travail (Dominique Chazal, Thomas Vigier, Pierre Pugin, Bruno Fautrez) ainsi que Valérie Fontanieu (statisticienne, INRP), qui a confectionné l'interface nécessaire entre la base et Pajek, et Martine Rémond (IUFM de Créteil, INRP) pour son aide précieuse dans l'analyse du matériau.

2 Antonio Novoa et Martin Lawn, Fabricating Europe: The Formation of an Education Space, Dordrecht, Kluwer, 2002; Jenny Ozga et Rob Jones, "Travelling and Embedded Policy: The Case of Knowledge Transfer ", Journal of Education Policy, vol. 21, n ${ }^{\circ}$ 1, janvier 2006, p. 1-17; Martin Lawn et Bob Lingard, "Constructing a European Policy Space in Educational Governance: The Role of Transnational Policy Actors ", European Educational Research Journal, vol. 1, no 2, 2002, p. 290-307; Miriam Henry et al., The OECD, Globalization, and Education Policy, Oxford, Pergamon-Elsevier, 2001. 
transformations de la matrice théorique et idéologique de l'OCDE et de la naissance d'un programme international sur les indicateurs de l'éducation. Ils montrent la façon dont la Commission Européenne crée et stabilise des réseaux d'acteurs transnationaux n'appartenant pas aux cercles officiels pour faire valoir un certain nombre de ces directives et recommandations auprès des États-Membres. Les idées, les modèles et les instruments de l'expertise circulent d'une organisation internationale à une autre, entre les États, selon un processus d'emprunt réciproque et de transfert de connaissances. Nous nous sommes intéressés à ces réseaux internationaux d'experts qui participent à la conception d'instruments de mesure pour l'éducation. Nous étudions les dimensions cognitives de leur activité, c'est-à-dire les connaissances produites par l'expertise qui ne peuvent être dissociées d'une communauté de pratiques, d'un engagement mutuel au service d'un projet commun, d'un répertoire d'actions et de discours. Mais les développements de l'expertise s'appuient aussi sur des jugements et des affinités difficilement objectivables. La connaissance tacite constitue une part incontournable du travail des experts et des scientifiques en même temps qu'elle relève d'un travail de coordination par ajustement mutuel et d'un contrôle des pairs ${ }^{3}$. Ce principe du contrôle mutuel assure que chaque expert exerce une fonction de surveillance sur ses collègues et une fonction d'autorité pour les plus reconnus parmi la communauté. De proche en proche, une chaîne de voisinage étend les garanties de scientificité et le consensus entre les membres.

Comment rendre compte de la production de l'expertise internationale tout en objectivant les relations de proximité entre experts? L'une des réponses consiste à étudier leurs signatures scientifiques. Bien plus que l'inscription de noms dans un article, la signature manifeste un acte de travail individuel ou collectif témoignant des conventions propres à une communauté scientifique $^{4}$. Elle rend compte de la hiérarchisation des activités de

Michael Polanyi, The Tacit Dimension, London, Peter Smith, 1983.

David Pontille, La signature scientifique : une sociologie pragmatique de l'attribution, Paris, CNRS Sociologie, 2004. 
production de connaissances (les seniors signent moins que les juniors), elle est articulée avec d'autres tâches moins reconnues (enquête, confection d'instruments, usage de banques de données, etc.), elle peut être dissociée de l'écriture scientifique (on peut signer sans écrire). La signature fait exister un espace public de discussion où les connaissances sont soumises à la critique et à la controverse avant d'être certifiées. Dans ce processus de certification, les articles jouent un rôle essentiel puisqu'ils témoignent des interactions d'un milieu d'expertise mais aussi de phénomènes de reconnaissance entre pairs. Les signatures sont donc soumises à un travail de codification révélant les conventions d'un milieu professionnel, la distinction entre les auteurs principaux et auteurs secondaires, le monopole détenu par certains signataires en position de porte-parole, les relations réciproques de coopération entre membres. Les signatures caractérisent aussi des relations de pouvoir entre les experts: ceux qui détiennent l'autorité, les positions de subordination, la délégation de compétences, les marques de soumission.

Notre méthode consiste à étudier les processus de co-signatures d'articles entre deux ou plusieurs experts à travers leurs copublications ${ }^{5}$. Pour nous, une co-publication est la trace d'un travail collaboratif mais aussi la démonstration qu'il existe un lien entre deux experts, base de la construction d'un réseau plus large. De co-publications en co-publications, et de liens en liens, il est possible de montrer que les experts qui publient le plus sont en position de porte-parole dans une communauté scientifique donnée. Ce régime de la co-publication traduit non seulement l'appartenance à une même communauté de pratiques mais une proximité entre des personnes qui se côtoient, se rencontrent, partagent des expériences. Elles possèdent des valeurs et des normes communes. Les co-publications sont des preuves de ces relations informelles mais structurantes pour la production des connaissances scientifiques. Elles témoignent d'un savoir tacite

Monique Dalud-Vincent et Romuald Normand, «Analyse textuelle et analyse de Réseaux : exemple du traitement d'une base de données bibliographiques à l'aide des logiciels Alceste et Pajek ", Bulletin de Méthodologie Sociologique, $\mathrm{n}^{\circ} 109$, janvier 2011, p. 20-38. 
et d'une familiarité qui régissent la vie sociale des réseaux d'expertise. Ces réseaux sont analysés selon une dimension transnationale. Nous étudions les relations entre experts au sein de communautés épistémiques conçues comme des groupes de professionnels dotés d'une compétence reconnue dans un domaine scientifique et associés à une même entreprise dans la production de connaissances ${ }^{6}$. Une communauté épistémique n'est pas un réseau comme les autres dans la mesure où cette communauté se construit par rapport à un paradigme scientifique et qu'elle rassemble des spécialistes d'un domaine précis (des scientifiques, des experts, des consultants), lesquels partagent des idées et des croyances sur les relations de cause à effet, sur une même épistémologie (principes et causalités, tests de validité des savoirs) et sur une même stratégie d'entreprenariat politique pour diffuser les croyances internes parmi des lobbies et des décideurs politiques. Ces communautés épistémiques attirent à elles d'autres acteurs (parlementaires, fonctionnaires d'États et des grandes organisations internationales) et parviennent à atteindre un consensus dans un domaine d'expertise précis, ces savoirs étant ensuite diffusés et accaparés par d'autres acteurs.

Dans l'enquête, nous avons pris en compte quatre domaines d'expertise à l'échelle internationale correspondant à trois communautés épistémiques préalablement étudiées : les chercheurs de l'école efficace (school effectiveness), les économistes du capital humain, les spécialistes de la littératie dans les comparaisons internationales de résultats comme Pisa. Elles ont joué un rôle structurant dans la fabrication d'indicateurs à l'échelle nationale et internationale dans les politiques d'éducation. Elles partagent un socle méthodologique et épistémologique commun dans l'usage des tests afin de mesurer les compétences acquises par les jeunes et les adultes, le rendement de l'éducation, ou l'efficacité des organisations scolaires et des systèmes éducatifs ${ }^{7}$. La school effectiveness postule que les établissements scolaires peuvent être

6 Peter M. Haas, «Introduction, Epistemic Communities and International Policy Coordination ", International Organization, n 46, 1992, p. 1-35.

7 Romuald Normand, Gouverner la réussite scolaire. Une arithmétique politique des inégalités, Berne, Peter Lang, 2011. 
évalués selon différentes variables et indicateurs, et que les enseignants sont les premiers responsables de l'amélioration des résultats des élèves. Cette école de pensée a justifié la mise en œuvre de systèmes d'évaluation par les tests et les politiques d'obligation de rendre compte dans bon nombre de pays. La théorie du capital humain établit que la mesure des compétences cognitives constitue un bon indicateur de la qualité des systèmes éducatifs. En travaillant les données des comparaisons internationales, les économistes pensent obtenir une mesure pertinente de l'impact de la qualité de l'éducation sur la croissance économique. Ils s'appuient donc sur les épreuves de tests en littératie et numératie développés par les psychologues pour évaluer et comparer les connaissances et les compétences des jeunes comme des adultes.

Après la construction d'une banque de données, l'usage du logiciel Pajek ${ }^{8}$ nous a permis de spécifier les liens entre ces différents scientifiques et experts, et de faire émerger certaines caractéristiques de leur coopération interne à un champ scientifique ou à un domaine d'expertise donné, ou les relations externes qu'ils pouvaient entretenir avec d'autres experts/scientifiques ou des décideurs politiques travaillant pour des gouvernements ou d'autres institutions. En adoptant une démarche latourienne, nous avons fait l'hypothèse que science et politique n'étaient pas dans une relation discontinue mais qu'au contraire, la confection d'instruments et de méthodologies de la mesure à l'échelle internationale mobilisait un corpus de connaissances scientifiques et des principes normatifs portés par différents porte-parole ${ }^{9}$. Afin de stabiliser ces énoncés en les traduisant en référentiels des politiques éducatives, le recours à l'expertise

8 Andrej Mrvar Wouter De Noy et Vladimir Batagelj, Exploratory Social Network Analysis with Pajek, Cambridge, Cambridge University Press, 2005.

9 Romuald Normand, «L'école efficace ou l'horizon du monde comme laboratoire dans la construction des politiques d'éducation : de nouveaux rapports entre science et politique ", Revue Française de Pédagogie, n 154 , janv-févrmars 2006, p. 33-44; Romuald Normand, "School Effectiveness or the World as a Laboratory ", British Journal of Sociology of Education, vol. 29, $\mathrm{n}^{\circ}$ 6, novembre 2008, p. 665-676. 
permet de donner une légitimité et une force à la décision politique. La mise en évidence des réseaux d'experts autour de la mesure en éducation a servi à repérer ces processus de diffusion et de circulation des connaissances et des instruments, en même temps que les opérations de traduction du monde scientifique au monde politique. Nous avons cherché à repérer des configurations, des lieux et des moments de connexion entre différentes entités, qu'ils s'agissent d'individus ou d'institutions, en nous inspirant du cadre d'analyse programmatique développé par Bruno Latour ${ }^{10}$. Celui-ci propose de :

- Localiser le global : pour nous, il s'agissait d'établir les connexions continues qui menaient d'une interaction locale entre experts jusqu'aux lieux de la mise en action de cette expertise dans un réseau plus large, au prix d'une traduction assurée par un médiateur ou porte-parole. Cela nous obligeait à repérer les sites locaux et les institutions globales où s'élaborait l'expertise et où s'opéraient les connexions. De connexions en connexions, nous avions à apprécier les changements d'échelle introduits par le passage du local au global, et le rôle joué par certains centres de calcul dans la mise en relation de ces réseaux et la circulation de l'information.

- Redistribuer le local: en nous centrant spécifiquement sur la France, l'étude proposait d'analyser la nature des connexions qui s'étaient établies entre des sciences de gouvernement de l'éducation proche du ministère de l'Éducation nationale et les réseaux internationaux de l'expertise sur la mesure. Cet équipement métrologique des scientifiques et des responsables politiques dans un cadre national permettait de faire ressortir des interactions dépassant les frontières étatiques et de montrer des articulations fortes avec la communauté internationale. Nous étions intéressés par le rôle joué par certains experts dans la médiation opérée entre un centre de calcul local (la

$\overline{10}$ Bruno Latour, Changer la société. Refaire la sociologie, Paris, La Découverte, 2006. 
Direction de l'Évaluation et de la Prospective) et des institutions comme l'OCDE ou la Commission Européenne. Nous voulions identifier systématiquement des lieux de rencontre des experts et des décideurs politiques dans différents pays, lorsqu'ils participaient à la production de référentiels nouveaux.

- Connecter des espaces de calcul: une fois repérés les réseaux d'experts, la présence de porte-parole et de médiateurs, leur appartenance à différentes institutions, leurs rencontres sur différents sites, il s'agissait de caractériser les connexions établies non plus entre les individus eux-mêmes mais entre différents centres de calcul à l'échelle internationale. Nous souhaitions tester l'hypothèse selon laquelle la fabrication de l'expertise et de la métrologie internationales ne relevait pas seulement un jeu d'acteurs individuels mais qu'elle dépendait aussi de l'investissement des États-nations dans des dispositifs et des programmes transnationaux portés par des institutions ad hoc, publiques ou privées.

Nous ne pouvons pas présenter ici l'ensemble des résultats de cette recherche. Aussi, nous nous sommes limités à la présentation de la méthodologie et des résultats concernant les liens établis entre experts et porte-parole appartenant à différentes communautés épistémiques.

\section{Comment construire ces réseaux d'experts et scientifiques?}

Nous avons donc réfléchi à la méthodologie la mieux adaptée pour saisir la dynamique des configurations à l'œuvre et assurer une traçabilité des réseaux du local au global. Même si nous nous sommes inspirés de méthodes déjà éprouvées comme l'analyse des réseaux et les analyses bibliométriques ${ }^{11}$, l'objet de l'enquête

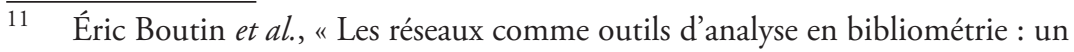
cas d'application : les réseaux d'auteurs ", Cahiers de la documentation, vol. 50, $\mathrm{n}^{\circ}$ 1, 1996, p. 3-13; Yves Gingras, La fièvre de l'évaluation de la recherche. Du mauvais usage de faux indicateurs, Note de recherche, Montréal, CIRST, UQAM, mai 2008; Hervé Rostaing, La bibliométrie et ses techniques, Sciences de la Société / CRRM, coll. "Outils et méthodes ", 1996; Johan Heilbron, "La bibliométrie, genèse et usages », Actes de la recherche en 
nous a contraints à opérer des choix dans les outils servant à la capitalisation des données, à leur traitement et à leur analyse.

Nous ne pouvions pas nous appuyer sur une banque de données normalisée en raison des biais qu'elle pouvait générer dans la représentation des réseaux. En effet, les données collectées dans les banques existantes proviennent souvent de sources officielles et publiées dans des livres, des revues, ou des rapports ce qui tend à sous-estimer l'influence de toute une littérature " grise " dans l'élaboration de l'expertise et de la prise de décision politique. Aussi, les acteurs et les institutions y sont présentées de manière évidente et naturalisée sans que soit interrogé le type de relations entre les acteurs, leur appartenance à une communauté épistémique, et leurs positionnements respectifs dans les sphères de la science, de l'expertise et de la politique. Ces revues de la littérature se révèlent, en outre, incapables de circonscrire des communautés épistémiques diversifiées tant par leurs disciplines de référence que par leur relations à des institutions internationales. Elles ne donnent pas à voir, ou très rarement, l'importance des programmes politiques internationaux de même qu'elles n'établissent pas de distinction entre les laboratoires universitaires et d'autres institutions de production des connaissances scientifiques comme par exemple des agences ou des think tanks. On peut aussi constater que la mise en présence des communautés scientifiques n'est pas seulement repérable à travers des publications communes mais aussi dans des lieux de rencontre qui ne sont pas mentionnés dans les revues de littérature: conférences internationales, groupes de travail opérationnels, clusters, etc. Pour finir, ces méthodes ne permettent pas de saisir la complexité des statuts et des positions des acteurs aux prises avec des identités multiples: une même personne peut être à la fois expert, chercheur, décideur politique.

Pour toutes ces raisons, la méthodologie a donc été itérative et elle s'est opérée selon plusieurs étapes.

sciences sociales, $\mathrm{n}^{\text {os }}$ 141-142, 2002, p. 78-79; Cyril Labbé et Ike Antkare, One of the Great Stars in the Scientific Firmament, Laboratoire d'information de Grenoble, 2010, [en ligne] http://www.pacte.cnrs.fr/IMG/pdf_IkeAntkareISSI. pdf, consulté le $1^{\text {er }}$ mars 2011. 
1.1. Première étape: établir une banque de données internationales pour repérer les individus et les réseaux

\section{Plateforme de stockage et de tri des données: Wikindx}

Notre choix s'est porté sur une plateforme en ligne utilisée par les documentalistes pour établir des références bibliographiques. Ce dispositif possédait l'avantage de permettre un travail à distance des membres de l'équipe de recherche tout en gérant les doublons (une référence déjà intégrée dans la base est repérée et signalée).

Cette banque de données, grâce à ses fonctionnalités standards, permettait aussi d'opérer différents tris selon des variables bibliométriques, et d'établir ainsi des listes d'auteurs, de publications, ou de repérer d'autres occurrences (dates, concepts communs, etc.).

\section{Choix d'un moteur de recherche}

Pour les raisons déjà évoquées (éviter un état construit de la science...) mais aussi à cause des difficultés d'interface et de collecte à distance, les bases de données comme Eric et Francis n'ont pu être utilisées. Nous avons finalement choisi Google Scholar comme moteur de recherche afin d'approvisionner la banque de données Wikindx. Le format standard Bibtex a l'avantage de s'importer par " copier/coller " dans Wikindx ce qui permettait une saisie rapide et automatique. Il s'agissait de capitaliser le maximum de références bibliographiques (quelle que soit la langue utilisée) d'un auteur, en recensant aussi ses publications avec d'autres auteurs ${ }^{12}$.

\section{Définition d'une liste de mots clés et d'auteurs}

Des mots clés en français et en anglais ont servi à repérer les premiers individus afin de procéder ensuite, par extension, au

$\overline{12}$ Mais, bien sûr, nous n’avons pu échapper aux limites inhérentes à Google Scholar qui ne recense probablement pas (toutes) les publications anciennes ou encore les revues peu prestigieuses, voire les travaux d'experts qui gardent le plus souvent la forme de rapports internes. 
repérage d'autres individus qui leur étaient proches (en ayant co-publié avec les premiers). La liste des premiers mots a été la suivante : ability test, accountability, achievement, added value, assessment, attainment test, benchmarking, comparison, effectiveness, equity, evaluation system, evidence based, governance, human capital, improvement, input, leadership skills, measurement, output, performance, pisa, quality, results-based management, score, social indicators, standard, technical skill. Le travail s'est donc fait par "boule de neige " partant de mots clés mais il a aussi été réalisé à partir d'auteurs directement concernés par leurs publications et connus de l'équipe (par exemple, pour les travaux français, les chercheurs de l'Irédu ont permis de commencer le réseau avec des personnes comme Marie Duru-Bellat). De ces auteurs, on obtenait des publications et du même coup des co-auteurs et de nouveaux mots clés à partir desquels, on pouvait étendre nos recherches, etc. La liste des mots faisait donc grandir la liste des auteurs et la liste des auteurs faisait grandir la liste des $\operatorname{mots}^{13} \ldots$

1.2. Deuxième étape : des co-publications à l'établissement de liens entre les membres d'une même communauté épistémique

Une fois les références bibliographiques introduites dans la banque Wikindx, les données ont été importées et traitées dans le logiciel Pajek à partir des co-publications. Pour cela, nous avons construit une relation (qui prend la forme d'un graphe dans Pajek) entre les auteurs recensés. Cette relation est la suivante: il existe un lien entre deux auteurs dès lors que ces deux auteurs ont publié ensemble un ouvrage, un article... autrement dit, deux auteurs sont reliés (dans les deux sens) s'ils sont ensemble dans une même référence bibliographique. Bien sûr, si plus de deux auteurs ont écrit un article ou un ouvrage ensemble, ils admettent autant de liens deux à deux entre eux qu'il y a de

13 Un tel réseau n'a, bien sûr, pas de limite et il est difficile d'arrêter un tel travail. Nous avons stoppé les recherches lorsqu'il nous est apparu que les auteurs et les références apparaissant étaient déjà dans la base et que l'on commençait à " tourner en rond ». Les délais obtenus pour le projet étaient un deuxième argument même s'il était moins scientifique. 
couples possibles d'auteurs. Pajek permet alors de représenter l'ensemble des auteurs et des liens de co-publications. Il permet aussi de montrer des sous-réseaux (pour une tranche d'années, pour un auteur, etc.). Ainsi, chaque co-publication permettait d'établir un lien de proximité entre un auteur/chercheur et un ou plusieurs de ses collègues. Au-delà du repérage de relations interindividuelles, ont été établies des configurations de liens entre les membres d'une même communauté épistémique, avec des auteurs en position centrale (des porte-parole ou médiateurs) et des auteurs en position périphérique (parce que possédant moins de légitimité dans le champ scientifique). Le logiciel Pajek a permis d'accéder à une représentation graphique des différents réseaux et de dresser une première cartographie de ces communautés épistémiques.

\section{Premiers résultats de l'enquête}

\subsection{Quelques résultats statistiques d'ensemble}

La base contient 3500 auteurs et 5300 références. Le graphique $1^{14}$ permet de voir, malgré les imperfections et limites de Google Scholar et le travail relativement artisanal que nous avons effectué pour repérer les références, que le champ des travaux qui nous intéressent progresse nettement et rapidement du point de vue du nombre des publications notamment à partir des années 1980. Les premiers travaux repérés datent des années $1920^{15}$. Comme on pouvait le penser, plus rares sont les publications écrites à 4 auteurs ou plus même si certaines parviennent à 11 auteurs.

$14 \quad$ Les graphiques sont reproduits à la fin de l'article.

15 C'est à partir de ce schéma que nous avons défini plusieurs périodes : avant 1980, de 1980 à 1989, de 1990 à 1994, de 1995 à 1999, à partir de 2000. 


\section{Tableau 1}

Liste des auteurs ayant le plus publiés (dans la base)

\begin{tabular}{|l|c|}
\hline Nom des auteurs & $\begin{array}{c}\text { Nombre de } \\
\text { publications }\end{array}$ \\
\hline Duru-Bellat & 190 \\
\hline Mingat & 163 \\
\hline Hanushek & 94 \\
\hline Demeuse & 76 \\
\hline Kirsch & 75 \\
\hline Layard & 73 \\
\hline Psacharopoulos & 69 \\
\hline Guthrie & 66 \\
\hline Mullis & 64 \\
\hline Meuret & 63 \\
\hline Levin & 60 \\
\hline Slavin & 60 \\
\hline Crahay & 59 \\
\hline Heyneman & 59 \\
\hline Krueger & 59 \\
\hline Wossmann & 55 \\
\hline Goldstein & 53 \\
\hline Reynolds & 52 \\
\hline Gorard & 50 \\
\hline Scheerens & 46 \\
\hline Schuller & 45 \\
\hline
\end{tabular}

Marie Duru-Bellat (Tableau 1) se retrouve en tête des auteurs ${ }^{16}$ ayant le plus publiés mais de nombreux auteurs non francophones sont très bien placés.

Du point de vue du type de publications, on trouve surtout des articles et des livres dans la base ( voir graphique 2).

On remarque aussi que les auteurs sont de plus en plus nombreux dans la base (voir graphique 3).

La moitié des auteurs que nous avons repérés est présent après 2000.

16 Les deux premiers auteurs, Marie Duru-Bellat et Alain Mingat, marquent une très nette différence avec les autres auteurs. Ces positions doivent être relativisées par le fait d'un travail de recherche plus approfondi concernant les auteurs français et leurs co-publications. Nous souhaitions, en effet, connaître et objectiver du mieux possible le réseau « interne » à la France et ses liens avec les autres pays notamment européens. 


\subsection{Résultats obtenus à partir des co-publications}

Une liste des auteurs (Tableau 2) les plus fréquents en termes de co-publications a d'abord été établie.

Tableau 2

Auteurs ayant le plus co-publiés avec les autres (les liens multiples avec un auteur sont comptés une seule fois)

\begin{tabular}{|l|c|l|c|}
\hline \multicolumn{1}{|c|}{ auteur } & $\begin{array}{c}\text { nombre d'auteurs } \\
\text { ayant co-publiés }\end{array}$ & \multicolumn{1}{|c|}{ auteur } & $\begin{array}{c}\text { nombre d'auteurs } \\
\text { ayant co-publiés }\end{array}$ \\
\hline \hline mingat & 65 & bosker & 39 \\
\hline krueger & 60 & valenzuela & 38 \\
\hline guthrie & 59 & creemers & 37 \\
\hline layard & 49 & wohlstetter & 36 \\
\hline smith & 49 & martin & 36 \\
\hline mullis & 48 & reynolds & 35 \\
\hline crahay & 46 & fuchs & 35 \\
\hline demeuse & 45 & schuller & 35 \\
\hline kirsch & 43 & schleicher & 35 \\
\hline thomas & 41 & feinstein & 35 \\
\hline hanushek & 41 & goldstein & 34 \\
\hline bennett & 41 & peterson & 34 \\
\hline duru-bellat & 40 & mcgaw & 34 \\
\hline williams & 40 & gray & 33 \\
\hline brown & 40 & tuijnman & 32 \\
\hline anderson & 40 & jenkins & 32 \\
\hline & & levin & 32 \\
\hline
\end{tabular}

Nous avons pu ensuite mettre en évidence différentes configurations d'acteurs correspondant à différentes communautés épistémiques (voir graphique 4).

Une première analyse rapide montrait la présence de porteparole mobilisant un grand nombre de liens autour d'eux: Andreas Schleicher, Marc Demeuse, Éric Hanushek, Noberto Bottani, Jaap Scheerens, Gilbert de Landsheere, Ina V. S. Mullis, Irving Kirsch, des auteurs dont nous connaissions par ailleurs l'importance des travaux scientifiques ou leur rôle d'expert dans leur domaine respectif. Apparaissaient aussi nettement des réseaux précis correspondant à la school effectiveness anglaise 
(autour de David Reynolds), à la school effectiveness néerlandaise (autour de Jaap Scheerens), aux économistes du capital humain (autour d' Éric Hanushek), à la school effectiveness belge (autour de Gilbert de Landsheere ou Marc Demeuse), aux travaux sur les comparaisons internationales de résultats (autour de Ina V. S. Mullis et Irving Kirsch), alors que Noberto Bottani et Andreas Schleicher, experts auprès de l'OCDE, jetaient des ponts entre ces différents réseaux.

\subsubsection{Différentes communautés}

En utilisant le logiciel Alceste ${ }^{17}$ qui permet de mener une analyse textuelle de l'ensemble de la base (fichier contenant toutes les références bibliographiques), nous avons pu extraire quatre souspopulations ${ }^{18}$ d'auteurs significatifs en fonction des mots particulièrement présents dans leurs références. En nous focalisant alors plus précisément sur chacune de ces quatre parties de la cartographie internationale, il était possible grâce à Pajek de spécifier les différents sous-réseaux d'experts correspondant à une communauté épistémique spécifique (voir graphique 5).

Des liens apparaissaient nettement entre David Reynolds (l'un des principaux fondateurs de ce mouvement de recherche en Angleterre) et d'autres chercheurs, notamment David Hopkins, chercheur et expert reconnu qui a exercé d'importantes responsabilités comme David Reynolds auprès du département de l'éducation britannique, mais aussi Pam Sammons, Louise Stoll,

17 Max Reinert, «Une méthode d'analyse des données textuelles et une application : Aurélia de G. de Nerval ", Bulletin de méthodologie sociologique, $\mathrm{n}^{\circ}$ 26, 1990, p. 24-54; Max Reinert, "Alceste, une méthode statistique et sémiotique d'analyse de discours. Application aux Rêveries du promeneur solitaire ", Revue française de psychiatrie et de psychologie médicale, tome V, vol. 49, octobre 2001, p. 32-36; Max Reinert, Le glossaire de la méthode Alceste - La terminologie utilisée avec des informations techniques, CNRS-Printemps, Université de Versailles-Saint-Quentin en Yvelines, 2001.

18 Un traitement plus récent (voir Monique Dalud-Vincent et Romuald Normand, op. cit.) de la base plus complète a permis d'obtenir cinq souspopulations mais les deux analyses restent globalement cohérentes : ici une seule classe concerne les économistes du capital humain alors que l'analyse plus récente la décompose en deux classes (les économistes du capital humain liés à la Banque Mondiale se détachent des autres économistes). 
Peter Mortimore, ou David Cuttance ayant participé aux développements de cette mouvance et à son rapprochement avec les décideurs politiques anglais. Des liens existaient aussi entre cette school effectiveness anglaise et son jumeau néerlandais représenté ici par Bert Creemers, Jaap Scheerens et Roel Bosker de l'université de Twente. D'autres chercheurs apparaissaient davantage en périphérie comme Stephen Gorard (spécialiste de la mesure de l'équité), Caroline Gipps ou Harvey Goldstein (spécialiste de la méthodologie de la mesure et critique vis-à-vis de certaines dérives de la school effectiveness) (voir graphique 6).

Apparaissent ici deux réseaux distincts, l'un français et l'autre belge, dont l'unique lien est assuré par Denis Meuret, chercheur à l'Irédu, lequel a occupé quelque temps une position de responsable de l'évaluation au ministère (Direction de l'Évaluation et de la Prospective-DEP). Celui-ci est en relation avec Marc Demeuse (spécialiste de la construction d'indicateurs d'équité des systèmes éducatifs) et Walo Hutmacher, avec lequel il a travaillé, aux côtés de Norberto Bottani (pour le projet Ines : construction des indicateurs internationaux de l'enseignement pour l'OCDE). Marc Demeuse est lui-même en relation avec Dominique Lafontaine, Marcel Crahay, et Christian Monseur, chercheurs au Laboratoire de pédagogie expérimentale de Liège, une entité créé par Gilbert de Landsheere, spécialiste de l'évaluation et éminent expert auprès des grandes organisations internationales. Aletta Grisay, spécialiste reconnue de la school effectiveness, a effectué plusieurs études pour le compte de la DEP aux côtés de Denis Meuret. Pierre Vrignaud a produit un rapport sur les indicateurs internationaux de l'enseignement avec Norberto Bottani pour le compte de la DEP.

À l'autre extrémité, on trouve les chercheurs de l'Irédu (Institut de Recherche en Économie de l'Éducation), spécialistes de la mesure de l'efficacité de l'école, et experts auprès de la DEP: Marie Duru-Bellat, Bruno Suchaut, Alain Mingat, Nathalie Mons, Jean-Pierre Jarousse, Pascal Bressoux. En périphérie apparaissent François Dubet, auteur d'un rapport sur le collège avec Marie Duru-Bellat mais n'appartenant pas à ce courant de 
recherches, ainsi que Catherine Marry et Annick Kieffer, ayant travaillé avec Marie Duru-Bellat sur des questions de genre. À noter, enfin, la présence aussi des économistes Marc Gurgand, Éric Maurin et Dominique Goux, proches des théories du capital humain. Toutefois, la recherche française dans son ensemble apparaît assez isolée quand on replace ce réseau dans la configuration d'ensemble de l'expertise internationale ${ }^{19}$.

Trois groupes précis ont pu être identifiés (voir graphique 7). Le premier groupe, autour de Georges Psacharopoulous, correspond à des économistes ayant travaillé sur les pays en développement pour la banque mondiale. Ceci explique pourquoi on trouve Alain Mingat (Irédu-France) aux côtés d'autres chercheurs. À noter que Georges Psacharopoulos est aussi expert auprès de la Commission Européenne (DG Éducation et Culture) au sein du réseau d'économistes européens de l'éducation spécialement créé par la DG pour la conseiller dans ses recommandations sur les politiques d'éducation. Un second groupe est réuni autour de James Heckman (prix Nobel), comme Pedro Carneiro expert lui aussi auprès de la Commission Européenne. Un troisième groupe est repérable autour d'Éric Hanushek, lui aussi expert international reconnu dont le disciple, Ludger Wossmann, anime le réseau européen des économistes de l'éducation.

Irving Kirsch apparaît en position centrale, en relation avec Albert Tuijnman (voir graphique 8). Les deux experts ont travaillé à des comparaisons internationales sur la littératie des adultes pour l'OCDE. Albert Tuijnman a participé activement au programme Ines. On observe de proche en proche des liens avec Andreas Schleicher qui a assuré la coordination de l'enquête Pisa et Tom Schuller, tous les deux ayant exercé des fonctions importantes au sein de l'OCDE, auprès du Céri comme Barry McGaw, Albert Tuijnman, mais aussi Noberto Bottani ou Walo Hutmacher. John T. Guthrie, Peter B. Mosenthal et Lynn B. Jenkins ont aussi travaillé sur l'évaluation des compétences des adultes dans la littératie.

19 On peut probablement expliquer l'apparition de ce groupe par la langue (française) qui a créé une discrimination des données dans Alceste. 


\subsubsection{Réseau par période}

Avec Pajek, nous avons aussi cherché à établir une chronologie dans l'émergence des réseaux en optant pour le découpage suivant : avant les années 1980, puis les années 1980-1990, 19901995, 1995-2000, après 2000.

Avant les années 1980

Le graphique 9 montre les liens entre Éric Hanushek (économiste américain à la Hoover Institution) et Frédérick Mosteller, un des grands spécialistes des méthodes de mesure en éducation aux États-Unis, qui a introduit les essais contrôlés randomisés dans le cadre du projet Star, un programme de référence pour les économistes du capital humain (il montrait que la réduction de la taille des classes n'avait pas d'effet sur la réussite des élèves). Frédérick Mosteller a aussi contribué avec Patrick D. Monihyan à un séminaire de réflexion faisant suite au rapport de James Coleman et justifiant l'abandon des politiques d'éducation compensatoire aux États-Unis. À cette occasion, Éric Hanushek avait réalisé une étude pour Patrick D. Monihyan qui fut aussi le conseiller du président Richard Nixon. David C. Berliner est un chercheur qui a critiqué la pression du testing aux États-Unis et les politiques d'obligation de résultats. Georges Psacharopoulos est un économiste qui a travaillé pour la banque mondiale.

Les années 1980-1990

Un premier réseau (voir graphique 10) se structure autour d'Éric Hanushek et Dean Jamison, lesquels ont travaillé avec Ludger Wossmann sur des mesures économiques de la qualité de l'éducation. Stephen Heynemann, comme Ludger Wossmann, a travaillé sur les données de l'enquête Timss (troisième enquête internationale sur les mathématiques). Le réseau autour d'Alain Mingat regroupe les chercheurs français de l'Irédu notamment Jean-Pierre Jarousse et Marie Duru-Bellat, lesquels ont travaillé avec lui au cours de cette période sur les effets-maitre/classe/ établissement sur la réussite des élèves. La school effectiveness 
néerlandaise et britannique est aussi représentée avec respectivement Jaap Scheerens et David Reynolds et plus loin Louise Stoll, Peter Mortimore, Pam Sammons. On retrouve Harvey Goldstein et Caroline Gipps, ainsi que d'autres chercheurs critiques de la school effectiveness : Ian Hextall, Harry Torrance, et Patricia Murphy. Ina V. S. Mullis et Lynn B. Jenkins sont des spécialistes de l'évaluation de la littératie. Gene V. Glass, Barry McGaw sont des experts spécialistes de la méta-analyse, Barry McGaw a été directeur de l'éducation à l'OCDE. Henry M. Levin et Martin Carnoy sont des économistes de l'éducation influents au niveau international.

Les années 1990-1995

Desmond L. Nuttall est un spécialiste de la construction des indicateurs de qualité dans l'éducation, Harvey Goldstein et Caroline Gipps ont développé des réflexions méthodologiques sur l'évaluation et les comparaisons internationales de résultats, à distance de l'orthodoxie, comme Patricia Broadfoot (voir graphique 11). Le réseau composé de Thornstein Husen (le père des comparaisons internationales de résultats et l'un des fondateurs de l'IEA) d'Albert Tuijnman et de Noberto Bottani, avec T. Neville Postlewhaite (lui aussi un des fondateurs de l'IEA), représentent ceux qui ont travaillé pour l'OCDE dans la construction des indicateurs internationaux (projet Ines). Robert Slavin relève d'un autre courant de recherche (evidence-based research et le school improvement) visant à inventorier les bonnes pratiques pédagogiques permettant d'améliorer la performance des enseignants et la réussite des élèves. Autour de Irving Kirsch, Lynn B. Jenkins, Ina V. S. Mullis, on retrouve les experts des comparaisons internationales de résultats dans le domaine de la littératie, autour de Valenzuela et Georges Psacharopoulos les économistes proches de la banque mondiale, et d'Éric Hanushek les économistes du capital humain. 
Les années 1995-2000

On voit apparaître le réseau français de la school effectiveness avec Denis Meuret qui établit des liens avec Michael Schratz et John MacBeath (des spécialistes de l'auto-évaluation de l'établissement scolaire et de l'évaluation de la qualité de l'éducation). Au-delà des liens renforcés entre la school effectiveness britannique et néerlandaise, on observe une montée en puissance des experts du school improvement, les deux mouvements de recherche ayant développé des problématiques communes et renforcé leurs échanges. Cela explique par exemple la présence de David Hopkins et Alma Harris, deux importants représentants de ce courant de recherche. Sont mis en évidence quelques autres porte-parole: Tuijnman, Guthrie, Smith.

\section{Après 2000}

On repère un lien entre Marc Demeuse et Jaap Scheerens qui ont conçu tous les deux une batterie d'indicateurs pour la Commission Européenne, de même que des intrications plus fortes entre les paradigmes de la school effectiveness, du school improvement et du school leadership (voir graphique 13). À noter pour la partie française, l'émergence d'un acteur comme Jean-Richard Cytermann, ancien responsable de la DEP. Toutefois le réseau d'experts se densifie et son analyse nécessite plus de décompositions.

À partir de ces premiers repérages, pour les auteurs clés nous avons constitué une biographie permettant de déterminer les différentes fonctions occupées, les laboratoires, institutions d'appartenance, le déplacement des thèmes de recherche de l'auteur etc.. de manière à mener une analyse qualitative plus poussée.

\section{Conclusion}

La construction de réseaux scientifiques à partir des co-publications a révélé une première configuration composée de communautés épistémiques clairement identifiées et rattachés à un paradigme : des chercheurs de la school effectiveness, des économistes du 
capital humain, des psychométriciens spécialistes des comparaisons internationales de résultats. De même sont clairement apparus des individus occupant des positions de porte-parole d'un paradigme ou de médiateur entre différentes communautés. Dans différentes pays, ces communautés épistémiques ont contribué à une mise en ordre scientifique et technique au travers de normes et d'indicateurs orientant les politiques éducatives vers une obligation de résultats ${ }^{20}$. La mondialisation a toutefois imposé un changement d'échelle. C'est pourquoi nous avons étudié le rôle des institutions ou des agences internationales qui soutenaient la confection de ces instruments de mesure.

Une fois élaborés les réseaux épistémiques à partir des copublications entre chercheurs/experts sur la base d'un recensement systématique de leurs références bibliographiques, l'enquête s'est élargie à l'espace des connexions à d'autres acteurs, notamment les responsables politiques des institutions nationales ou supranationales. Ces données n'ont pas été présentées dans l'article. Nous avons mis en évidence les co-présences des chercheurs, des experts et des décideurs politiques, dans des forums publics ou semi-publics, au cours de conférences ou de séminaires débouchant sur la production d'un rapport, d'une recommandation ou d'une directive. Puis nous avons détaillé plus précisément les relations entre les différentes institutions où travaillaient décideurs politiques et experts à partir d'une cartographie en réseau des "centres de calcul " (agences, organisations internationales, ministères, associations, centres de connaissances, producteurs d'information statistique) qui concouraient à fabriquer de nouveaux instruments de mesure de l'éducation à l'échelle internationale.

Nous avons montré que légitimité des connaissances produites par l'expertise ne dépend pas seulement de la qualité intrinsèque de son épistémologie ou de sa méthodologie. Elle résulte d'un important travail de mobilisation de ressources en amont de la décision politique (financement, laboratoires, conceptions et

20 Stephen J. Ball, The Education Debate, Bristol, The Policy Press, 2008; Romuald Normand, Gouverner la réussite scholaire..., op. cit. 
expérimentations d'instruments), d'une capacité de l'expertise à se détacher du cadre national pour intégrer un programme transnational, de médiateurs (personnes ou institutions) capables de mettre en relation différents groupes sociaux, communautés épistémiques, ministères ou laboratoires de recherche, de l'alignement des savoirs experts sur un agenda politique au service d'une cause. C'est cet espace d'intéressement que l'OCDE a réussi à créer à travers son centre d'expertise (le Centre de Recherche sur l'Éducation et l'innovation) mais aussi des programmes transnationaux pour la conception d'enquêtes ou d'indicateurs mobilisant les États. Aujourd'hui, la Commission Européenne reprend et étend cette expertise pour soutenir sa Méthode Ouverte de Coordination en développant des indicateurs et des benchmarks dans le cadre de sa politique d'apprentissage tout au long de la vie ${ }^{21}$. Toutefois, au-delà de ses réseaux institutionnels, l'expertise internationale s'organise selon des " collèges invisibles ", c'est-à-dire des configurations informelles aux frontières floues où certains porte-parole représentent leurs collègues auprès des décideurs politiques ${ }^{22}$. De même la production des connaissances est multi-sites : elle se distribue dans des universités, des centres de recherche, des agences gouvernementales, des think tanks, des agences de consulting en s'appuyant aussi sur des réseaux de diffusion et de communication déterritorialisés. Cette redistribution des frontières entre science, expertise et politique à l'échelle internationale invite à réviser les outils et les méthodes de mesure de l'analyse sociologique qui se sont largement définies en France par référence à l'État et son système d'information statistique.

$\overline{21}$ Jean-Louis Derouet et Romuald Normand, "La mesure experte dans le gouvernement européen de la formation tout au long de la vie », dans Fabrizio Cantelli, Luca Pattaroni, Marta Roca et Joan Stavo-Debauge (dir.), Sensibilités pragmatiques. Enquêtes sur l'action publique, Berne, Peter Lang, 2009, p. 419435.

22 Diane Crane, Invisible Colleges: Diffusion of Knowledge in Scientific Communities, Chicago, The University of Chicago Press, 1972. 
Graphique 1

Répartition des références de la base de données selon l'année de publication et le nombre d'auteurs

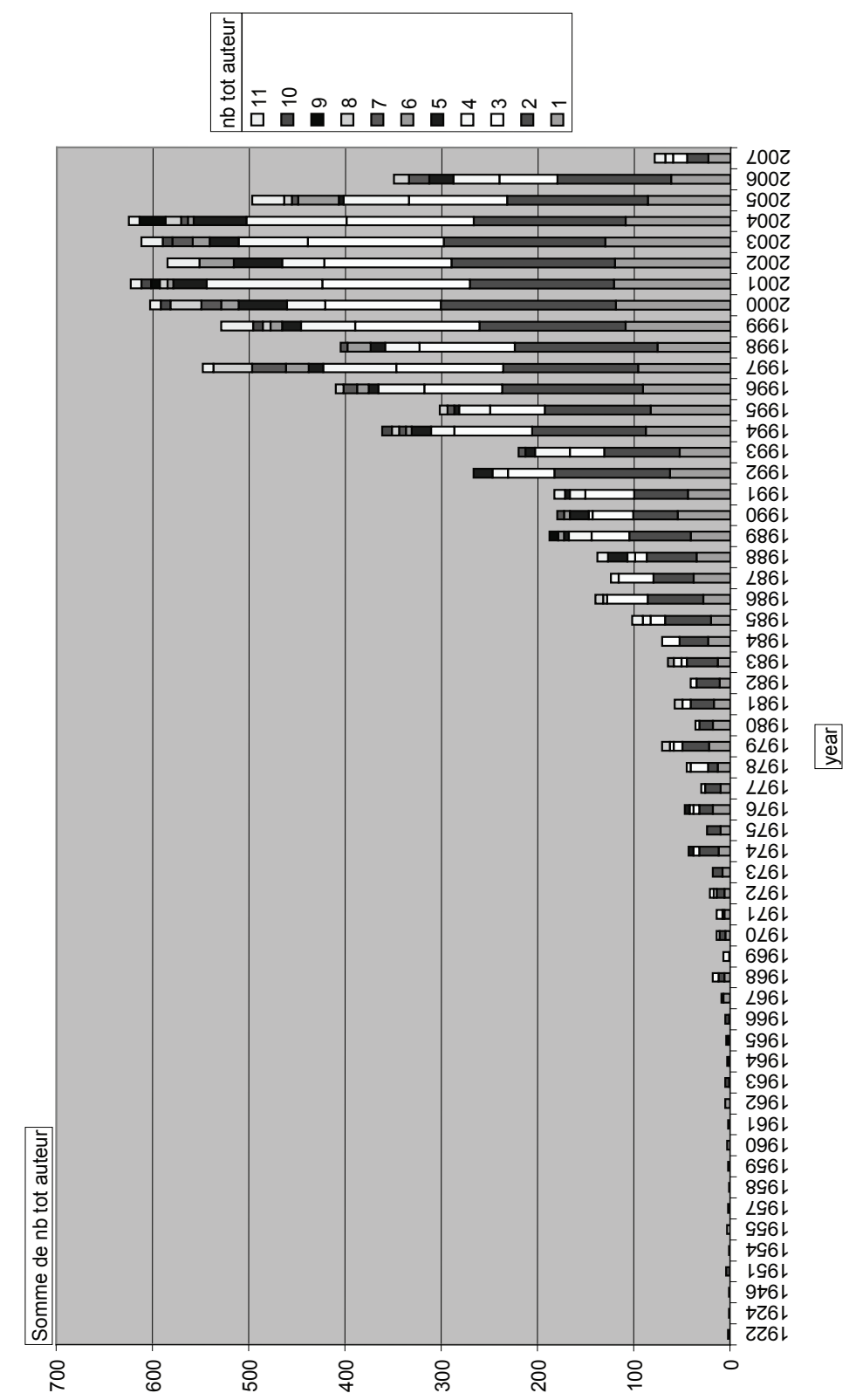


Graphique 2

Répartition des références de la base selon l'année et le type de publication
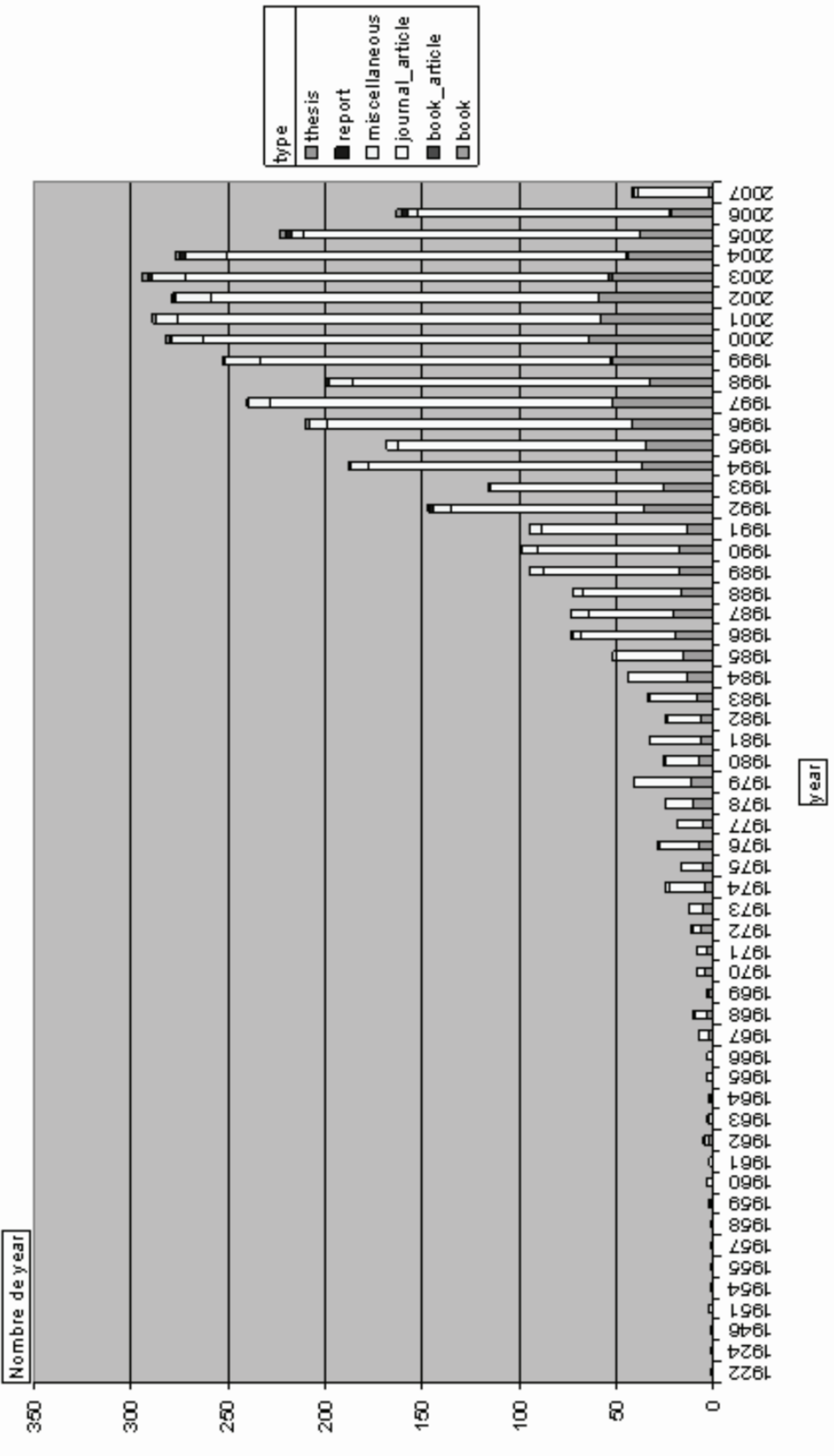
Graphique 3

\section{Répartition des auteurs de la base selon la période}

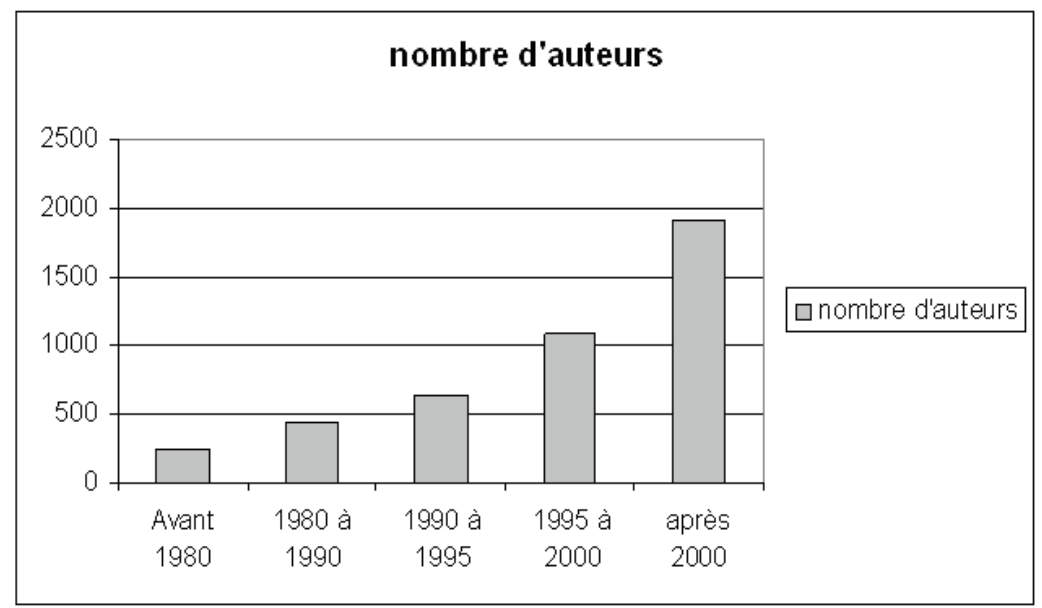


Graphique 4

Réseau des co-publications (extrait)

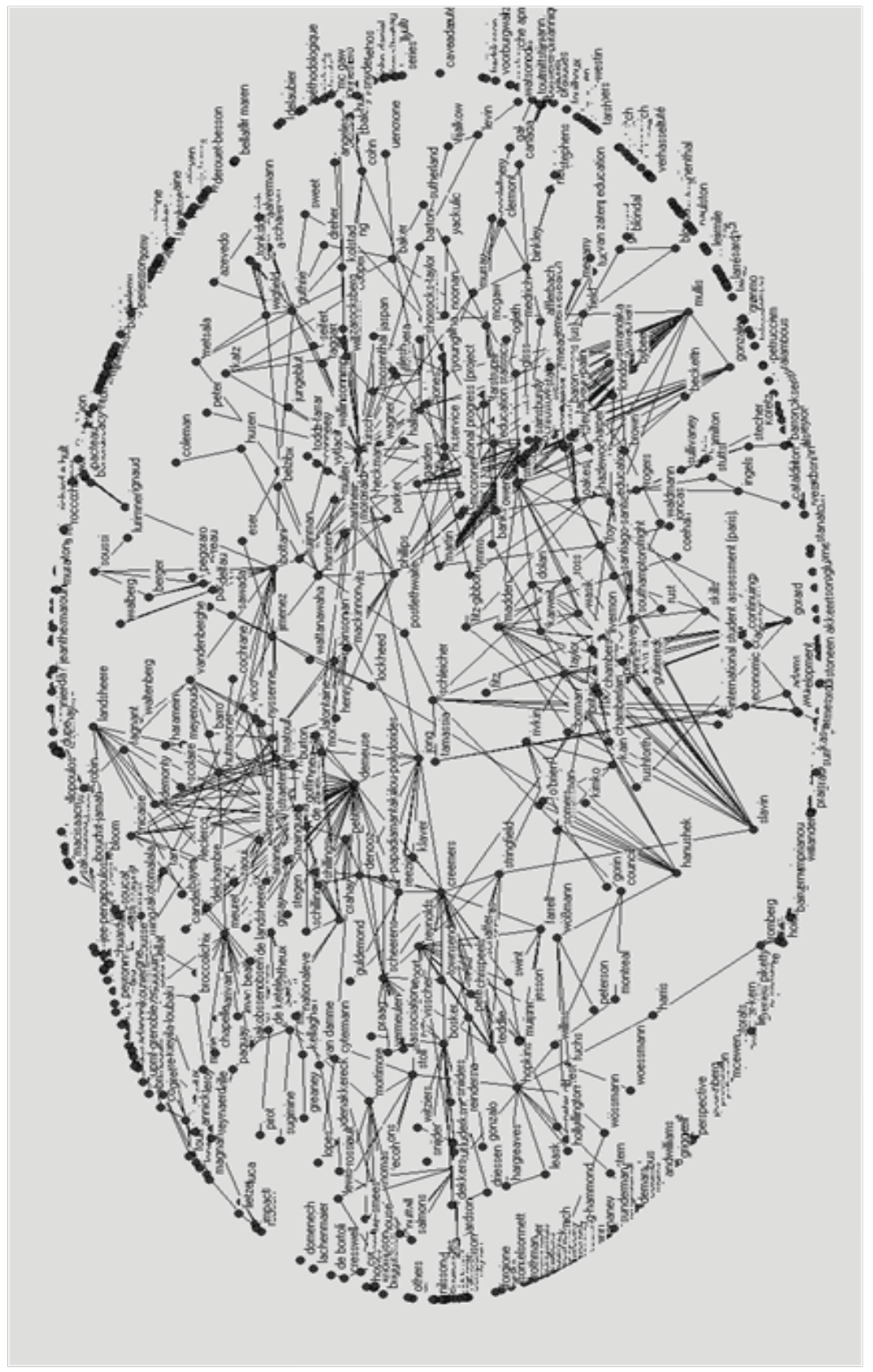


222 NPSS, VOLUME 6, NUMÉRO 2, 2011

Graphique 5

La school effectiveness anglaise et néérlandaise

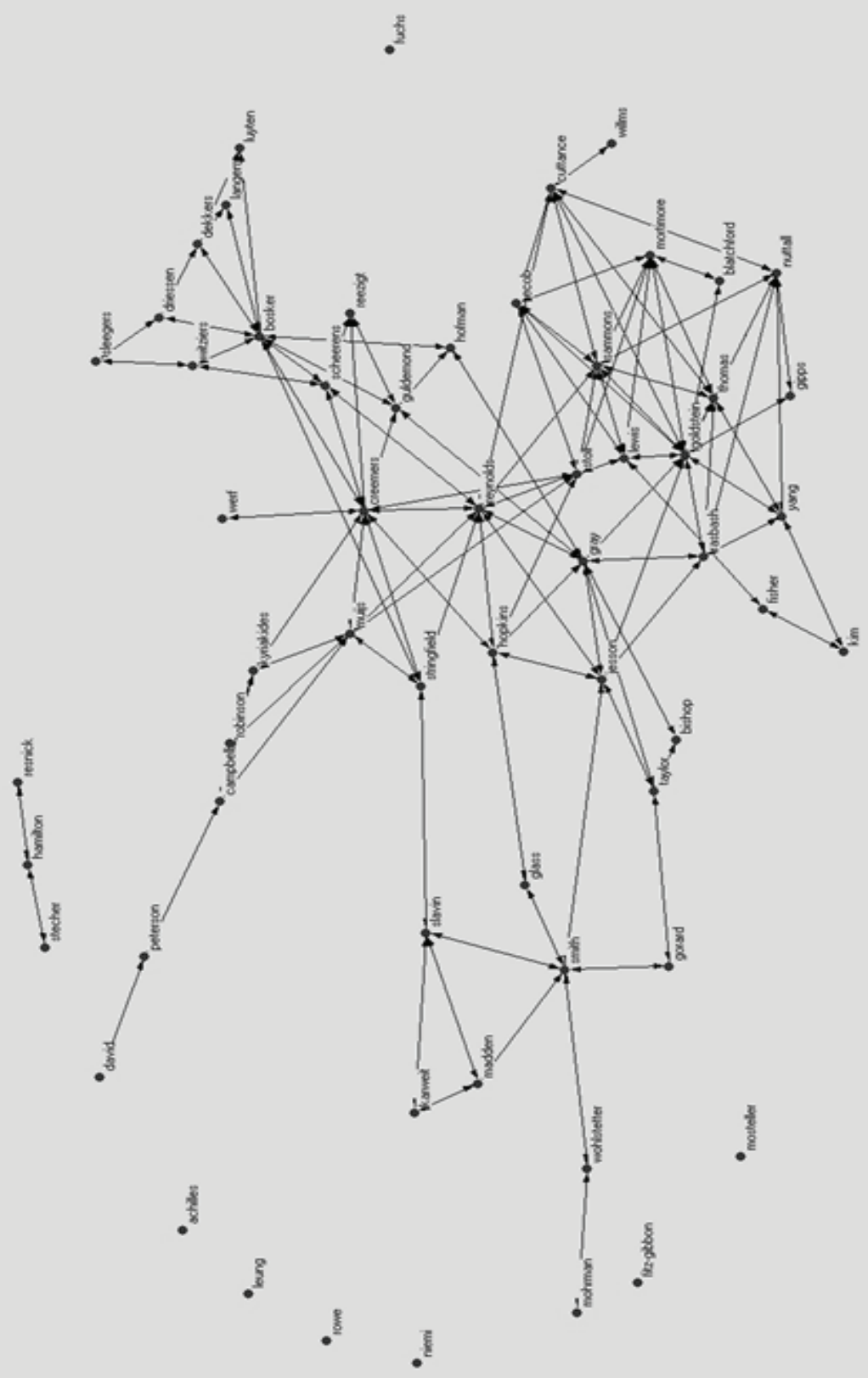


Graphique 6

\section{La school effectiveness franco-belge-suisse}

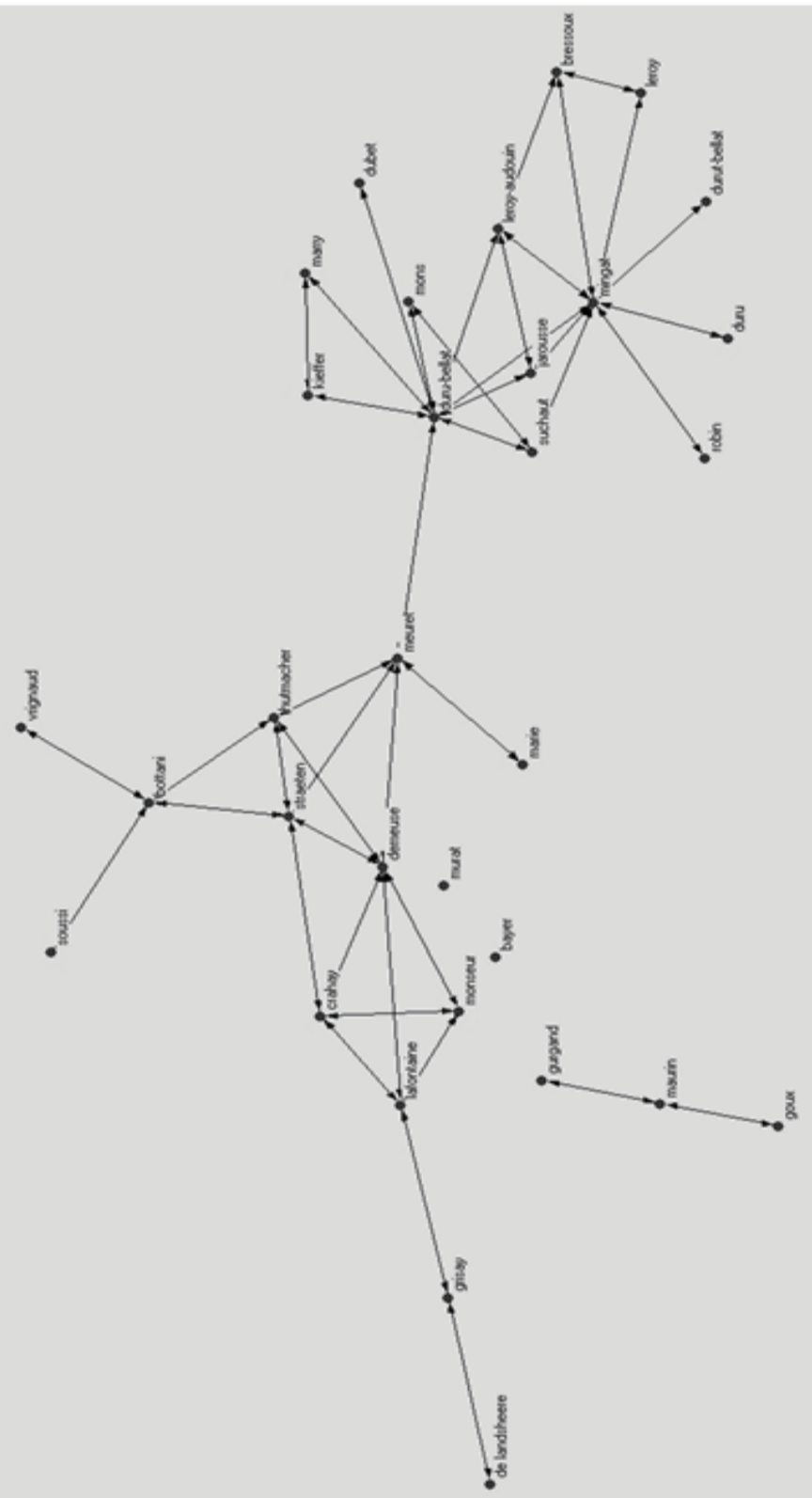


224 NPSS, VOLUME 6, NUMÉRO 2, 2011

Graphique 7

Les économistes du capital humain

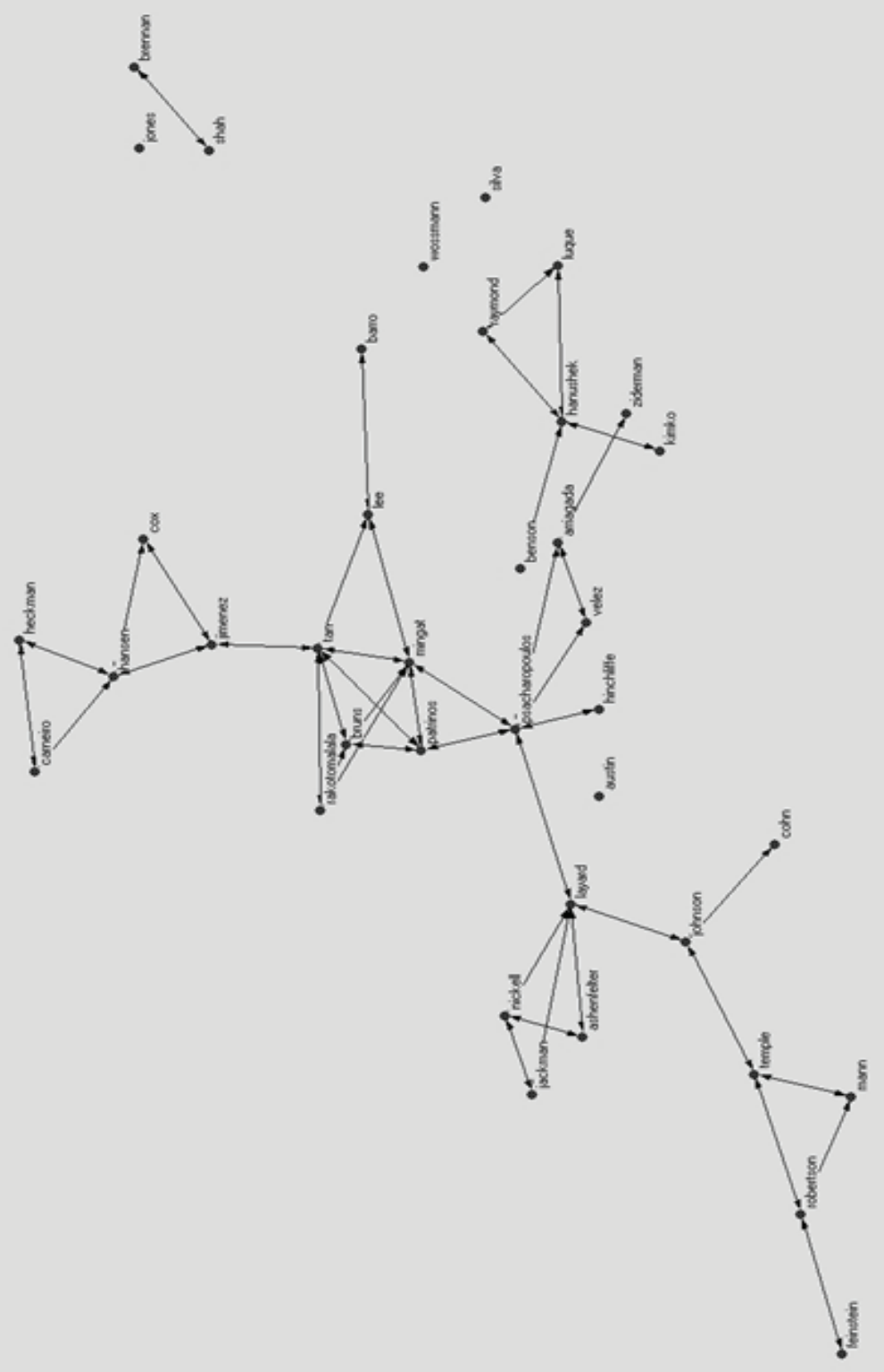


Graphique 8

Les experts de la littératie dans les comparaisons internationales de résultats

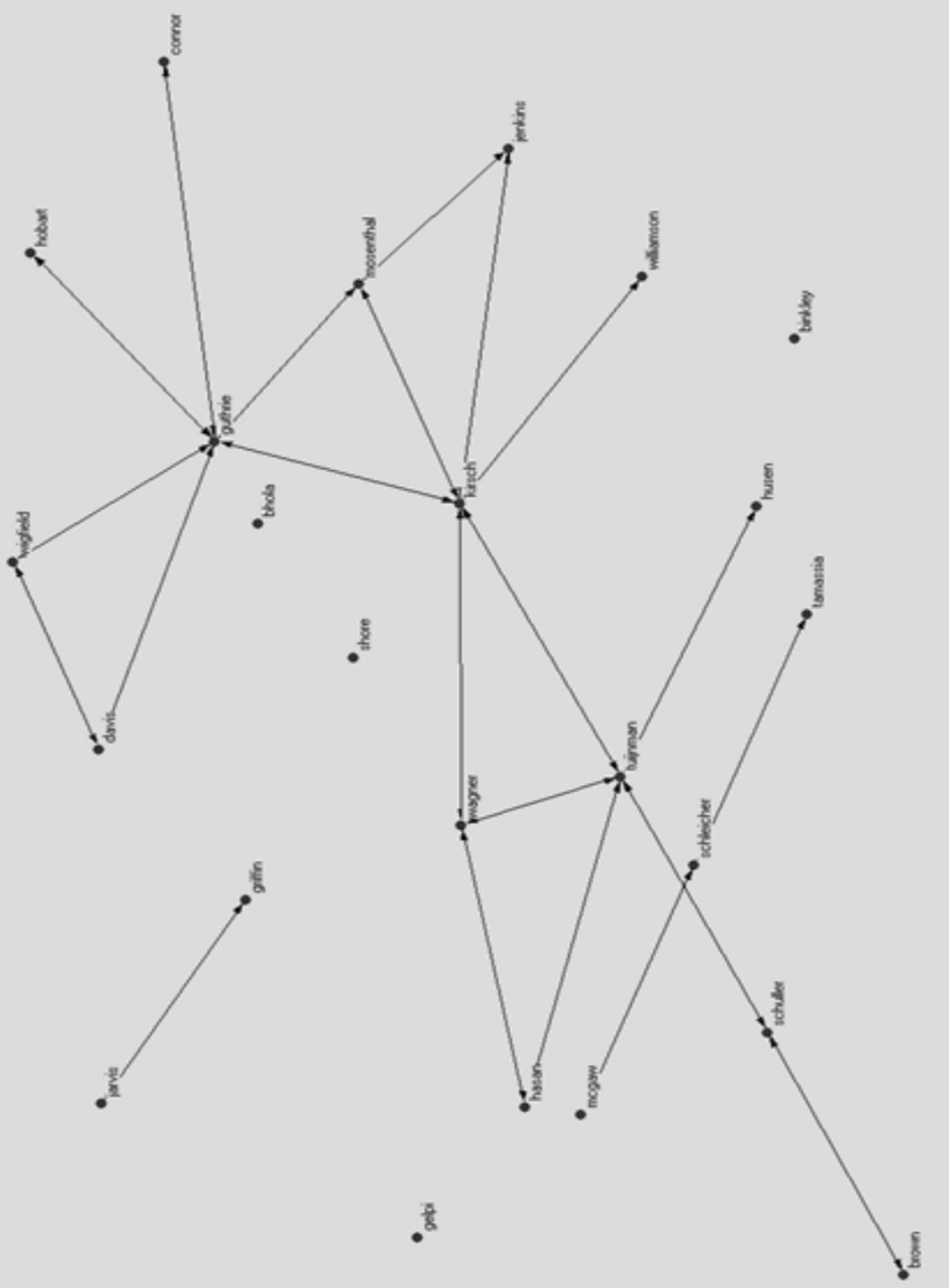


Graphique 9

Réseau des co-publications avant 1980 (composantes de 10 auteurs au moins)

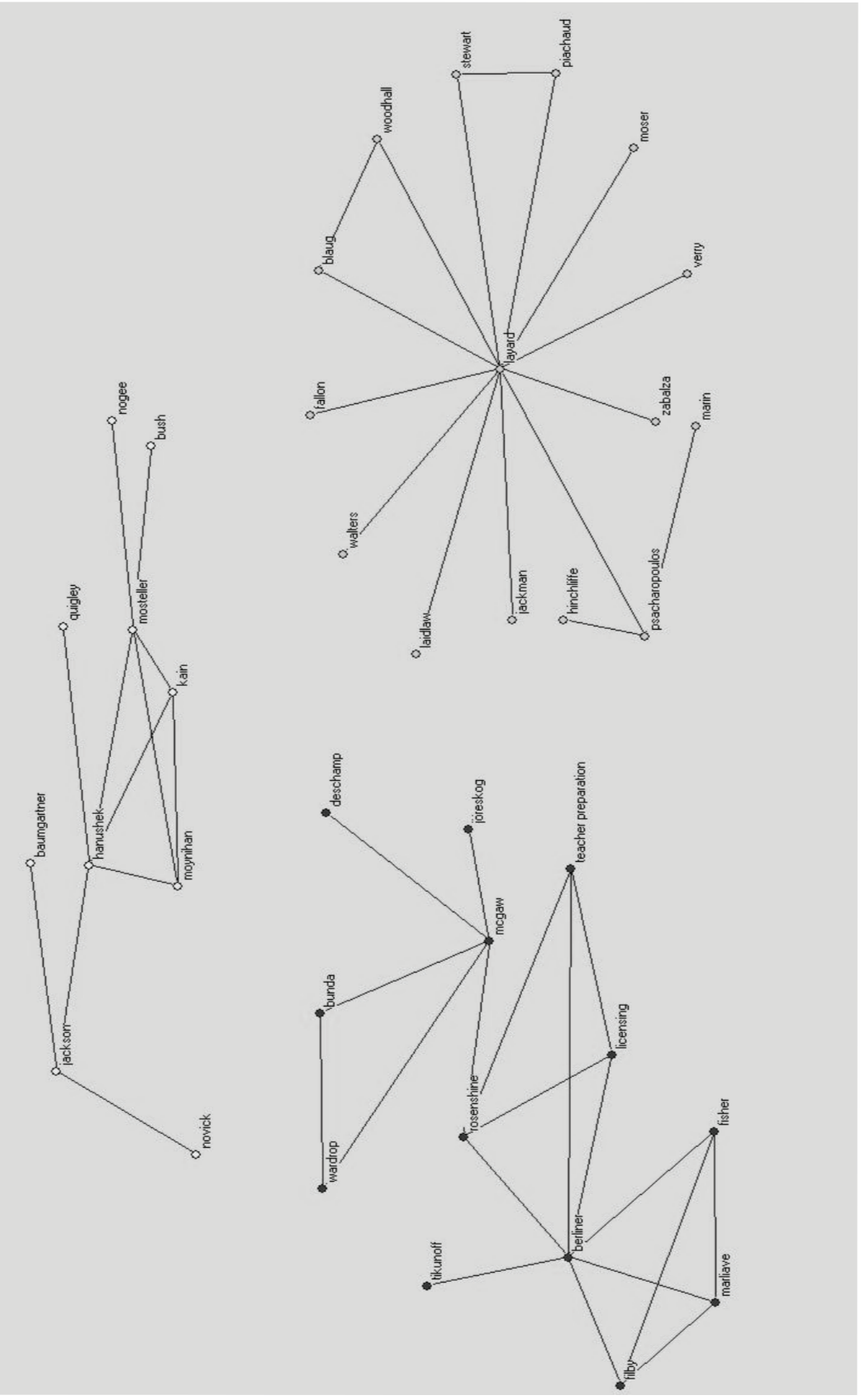


Graphique 10

Réseau des co-publications de la période 1980-1990 (composantes de 10 auteurs ou plus)

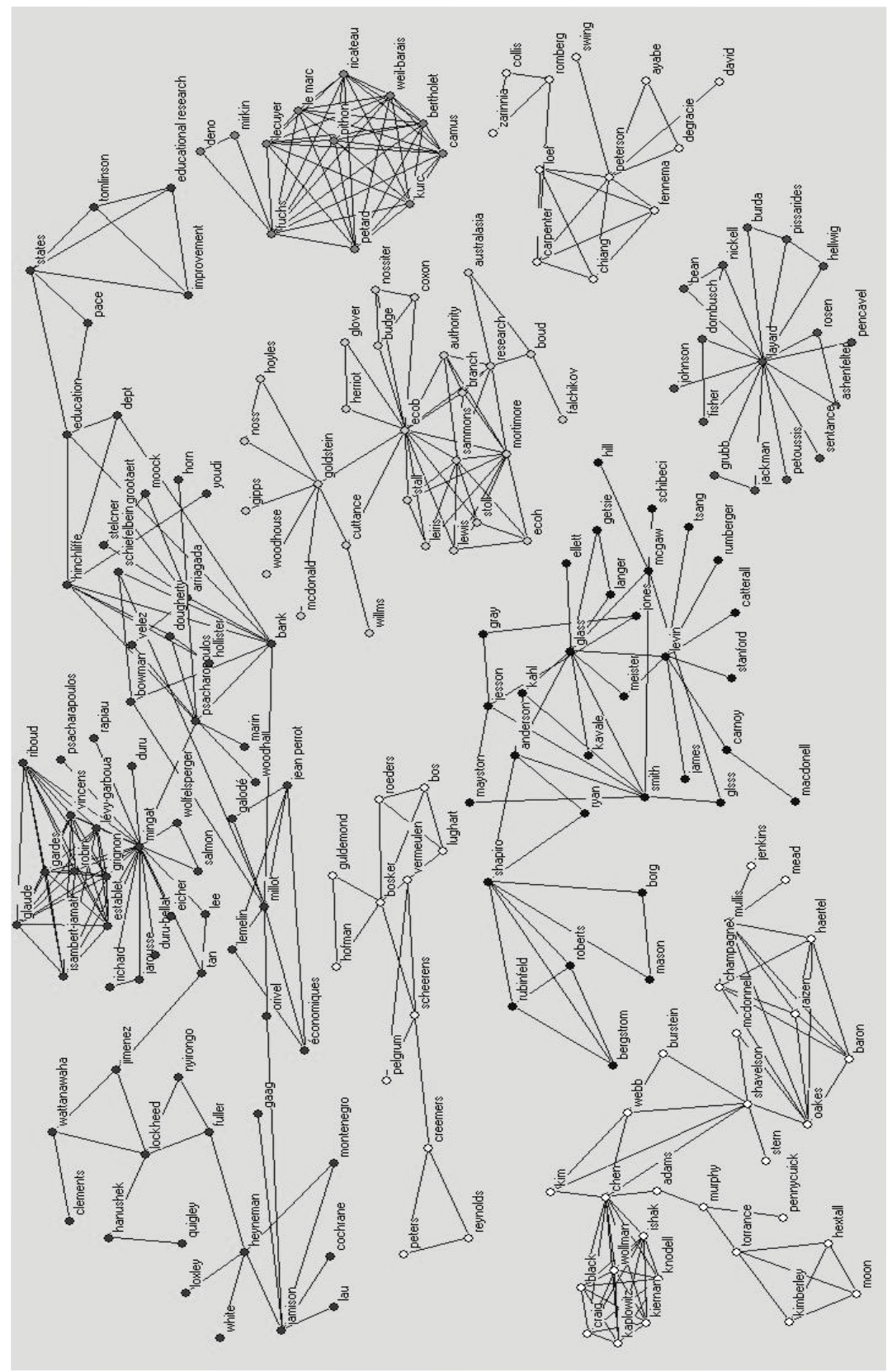


Graphique 11

Réseau des co-publications de la période 1990-1995 (composantes de 15 auteurs ou plus)

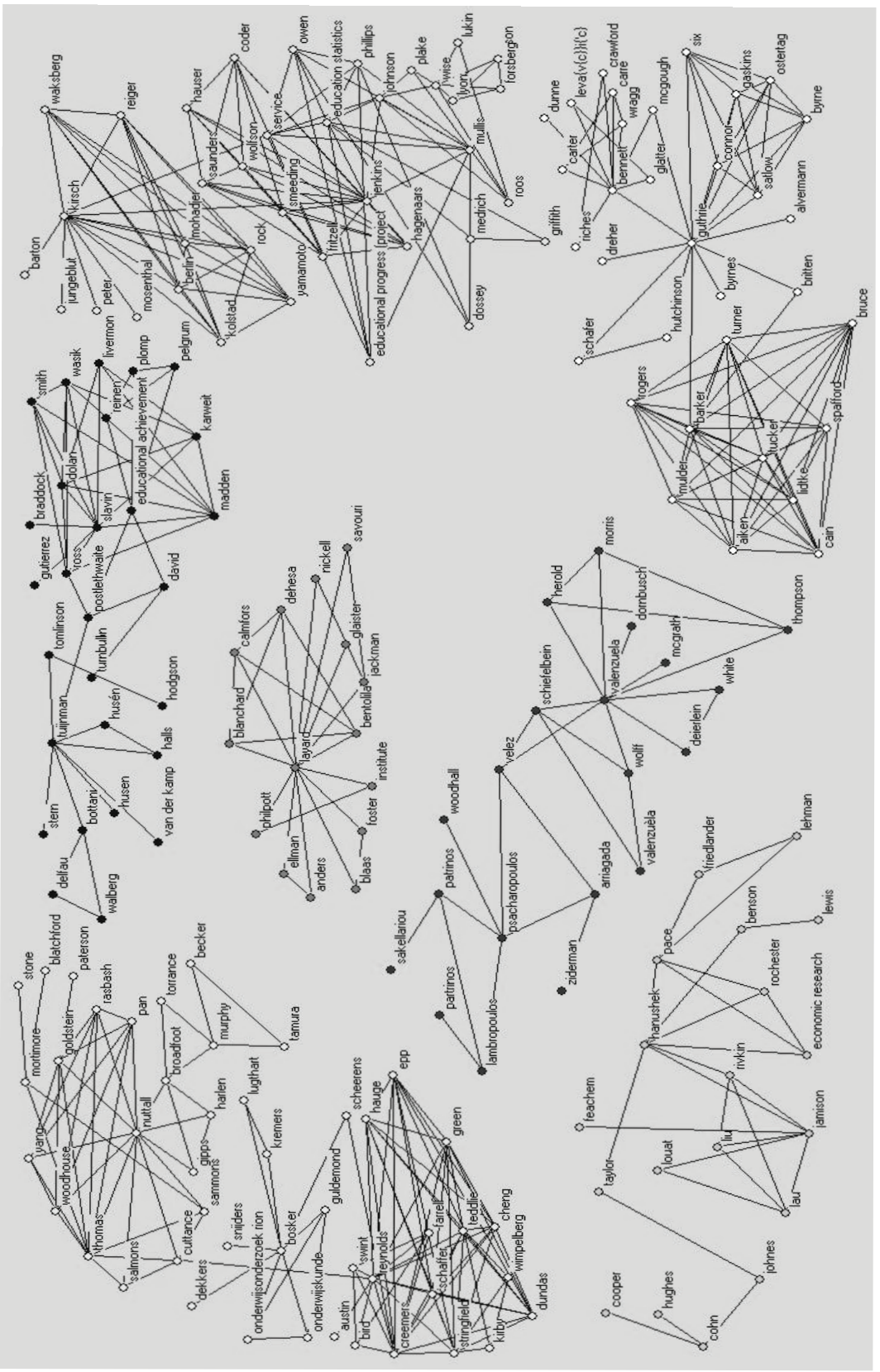


Graphique 12

Réseau des co-publications de la période 1995-2000 (composantes de 15 auteurs ou plus)

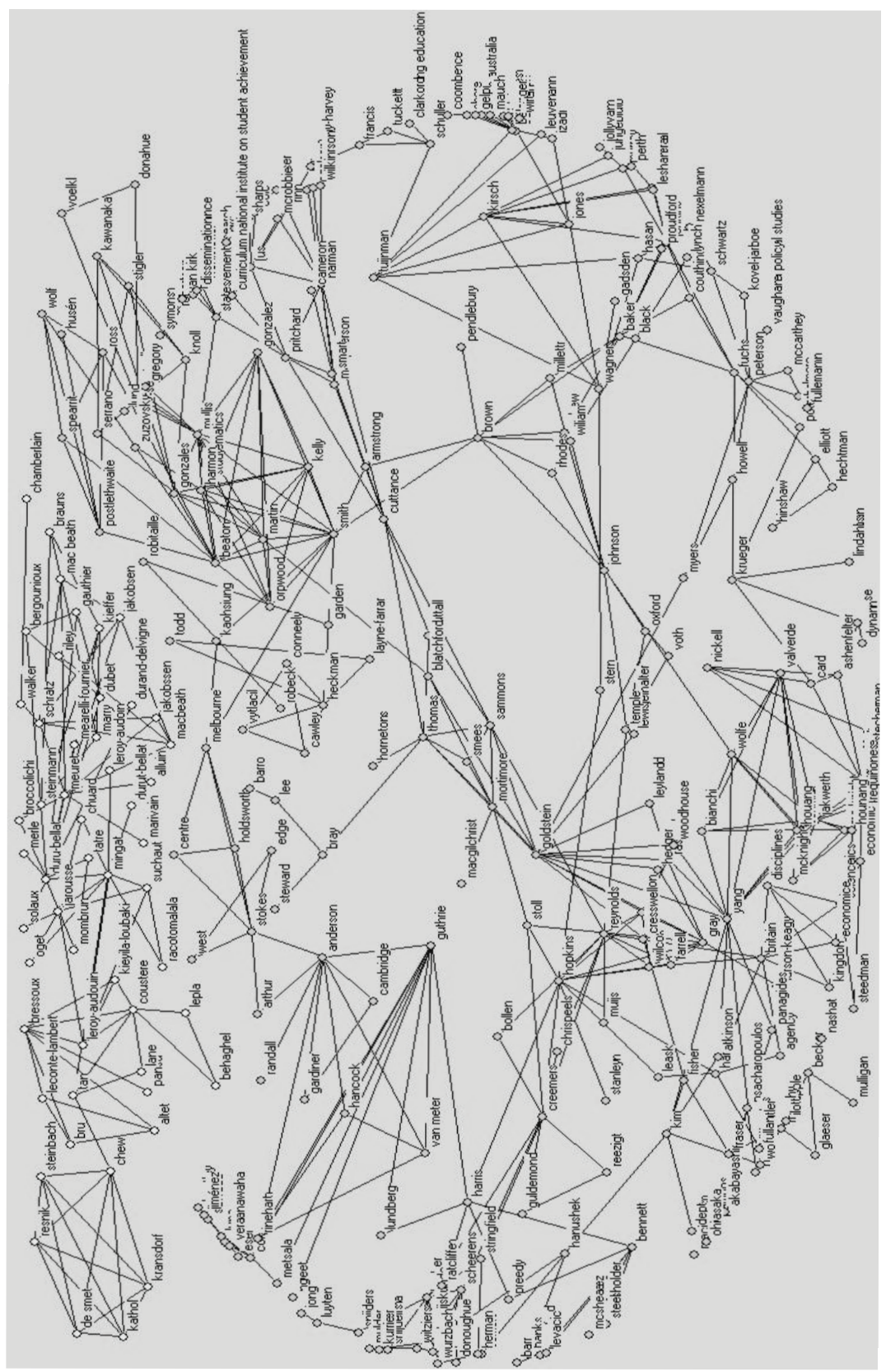


Graphique 13

Réseau des co-publications de la période après 2000 (composante la plus importante)

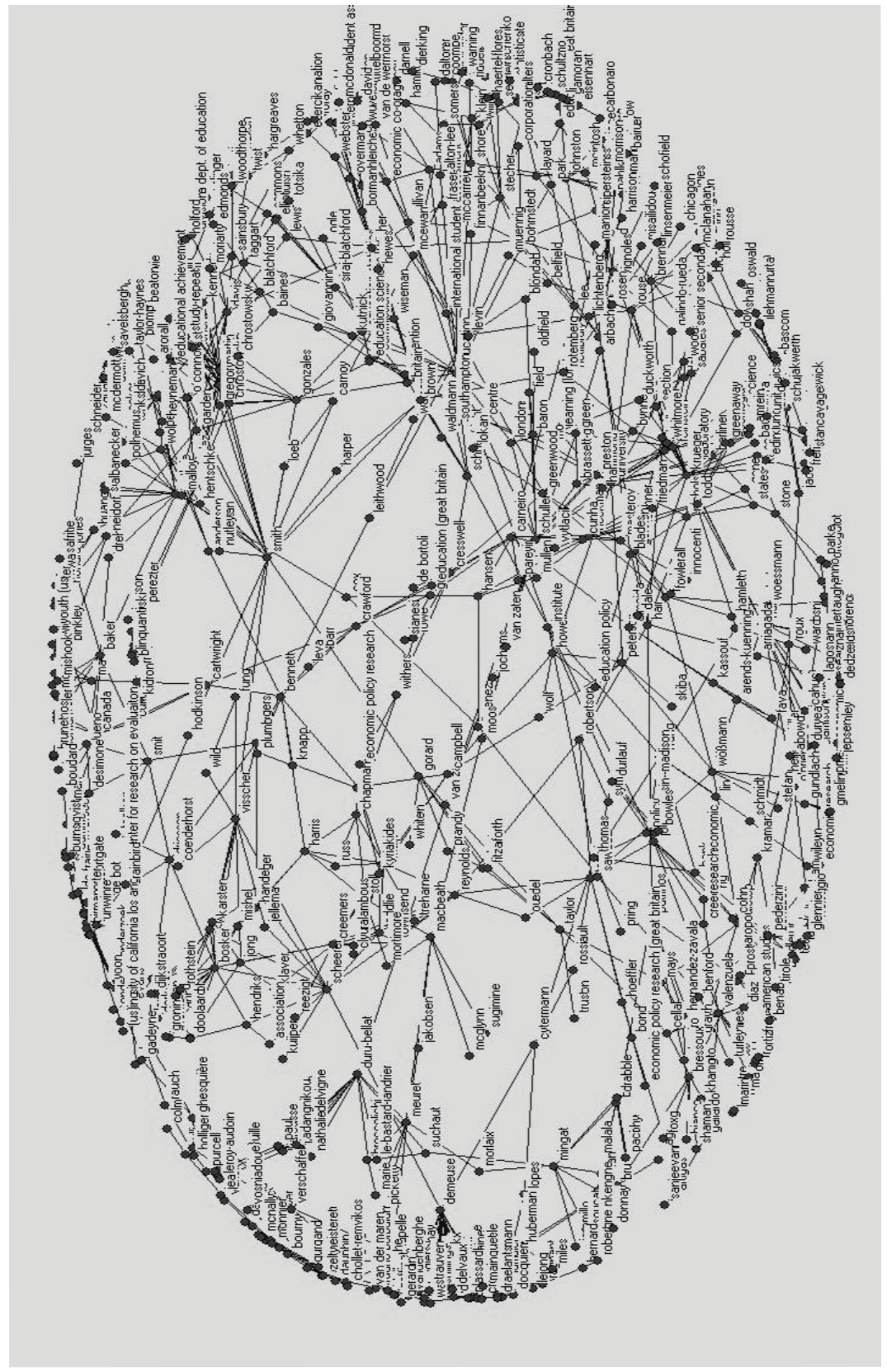




\section{Bibliographie}

Ball,Stephen J., The Education Debate, Bristol, The Policy Press, 2008.

Boutin, Éric et al. "Les réseaux comme outils d'analyse en bibliométrie : un cas d'application : les réseaux d'auteurs ", Cahiers de la documentation, vol. 50, n 1, 1996, p. 3-13.

Crane, Diane, Invisible Colleges: Diffusion of Knowledge in Scientific Communities, Chicago, The University of Chicago Press, 1972.

Dalud-Vincent, Monique et Romuald Normand, "Analyse textuelle et analyse de Réseaux : exemple du traitement d'une base de données bibliographiques à l'aide des logiciels Alceste et Pajek ", Bulletin de Méthodologie Sociologique, $\mathrm{n}^{\circ}$ 109, janvier 2011, p. 20-38.

De Noy, Andrej Mrvar Wouter et Vladimir Batagelj, Exploratory Social Network Analysis with Pajek, Cambridge, Cambridge University Press, 2005.

Derouet, Jean-Louis et Romuald Normand, "La mesure experte dans le gouvernement européen de la formation tout au long de la vie ", dans Fabrizio Cantelli, Luca Pattaroni, Marta Roca et Joan Stavo-Debauge (dir.), Sensibilités pragmatiques. Enquêtes sur l'action publique, Berne, Peter Lang, 2009, p. 419-435.

Gingras, Yves, La fière de l'évaluation de la recherche. Du mauvais usage de faux indicateurs, Note de recherche, Montréal, CIRST, UQAM, mai 2008.

Haas, Peter M., «Introduction, Epistemic Communities and International Policy Coordination», International Organization, $\mathrm{n}^{\circ} 46,1992$, p. 1-35.

Heilbron, Johan, "La bibliométrie, genèse et usages ", Actes de la recherche en sciences sociales, $\mathrm{n}^{\text {os }} 141-142,2002$, p. 78-79.

Henry, Miriam et al., The OECD, Globalization, and Education Policy, Oxford, Pergamon-Elsevier, 2001.

Knorr-Cetina, Karin, Epistemic Cultures: How the Sciences Make Knowledge, Cambridge/London, Harvard University Press, 1999.

Labbé, Cyril et Ike Antakare, One of the Great Stars in the Scientific Firmament, Laboratoire d'information de Grenoble, 2010; [en ligne] http://www.pacte.cnrs.fr/IMG/pdf_IkeAntkareISSI.pdf, consulté le $1^{\text {er }}$ mars 2011.

Lascoumes, Pierre et Patrick Le Galès (dir.), Gouverner par les instruments, Paris, Presses de Science-Po, 2004.

Latour, Bruno, La science en action, Paris, La Découverte, 1989. 
Latour, Bruno, Changer la société. Refaire la sociologie, Paris, La Découverte, 2006.

Lawn, Martin et Bob Lingard, "Constructing a European Policy Space in Educational Governance: The Role of Transnational Policy Actors», European Educational Research Journal, vol. 1, n ${ }^{\circ}$ 2, 2002, p. 290307.

Normand, Romuald, "L'école efficace ou l'horizon du monde comme laboratoire dans La construction des politiques d'éducation : de nouveaux rapports entre science et politique ", Revue Française de Pédagogie, no 154, janv-févr-mars 2006, p. 33-44.

Normand, Romuald, "School Effectiveness or the World as a Laboratory", British Journal of Sociology of Education, vol. 29, $\mathrm{n}^{\circ}$ 6, novembre 2008, p. 665-676.

Normand, Romuald, «Expertise, Networks and Tools of Government: The Fabrication of European Policy in Education", European Educational Research Journal, vol. 9, n 3, 2010, p. 408-423.

Normand, Romuald, Gouverner la réussite scolaire. Une arithmétique politique des inégalités, Berne, Peter Lang, 2011.

Novoa, Antonio et Martin Lawn, Fabricating Europe: The Formation of an Education Space, Dordrecht, Kluwer, 2002.

Ozga, Jenny et Rob Jones, «Travelling and Embedded Policy: The Case of Knowledge Transfer ", Journal of Education Policy, vol. 21, $\mathrm{n}^{\circ}$ 1, janvier 2006, p. 1-17.

Polanyi, Michael, The Tacit Dimension, London, Peter Smith, 1983.

Pontille, David, La signature scientifique : une sociologie pragmatique de l'attribution, Paris, CNRS Sociologie, 2004.

Reinert, Max, "Une méthode d'analyse des données textuelles et une application : Aurélia de G. de Nerval ", Bulletin de méthodologie sociologique, $\mathrm{n}^{\circ} 26,1990$, p. 24-54.

Reinert, Max, "Alceste, une méthode statistique et sémiotique d'analyse de discours. Application aux Rêveries du promeneur solitaire ", Revue française de psychiatrie et de psychologie médicale, tome V, vol. 49, octobre 2001, p. 32-36.

Reinert, Max, Le glossaire de la méthode Alceste - La terminologie utilisée avec des informations techniques, CNRS-Printemps, Université de Versailles-Saint-Quentin en Yvelines, 2001.

Rostaing, Hervé, La bibliométrie et ses techniques, Sciences de la Société I CRRM, coll. " Outils et méthodes ", 1996. 